\title{
Nephrology
}

\section{Clinical Trial of Vadadustat in Patients with Anemia Secondary to Stage 3 or 4 Chronic Kidney Disease}

\author{
Edouard R. Martin ${ }^{\mathrm{a}}$ Mark T. Smith ${ }^{\mathrm{b}}$ Bradley J. Maronic ${ }^{\mathrm{c}}$ Qing C. Zuraw ${ }^{\mathrm{c}}$ \\ Emil M. deGoma ${ }^{c}$ \\ a South Florida Nephrology Associates, Lauderdale Lakes, FL, ${ }^{b}$ Nephrology Associates, PC, Augusta, GA, and \\ 'Akebia Therapeutics Inc., Cambridge, MA, USA
}

\section{Keywords}

Anemia - Chronic kidney disease - Clinical trials .

Erythropoietin $\cdot$ Kidney disease

\begin{abstract}
Background: Therapeutic options for the treatment of anemia secondary to chronic kidney disease (CKD) remain limited. Vadadustat (AKB-6548) is an oral hypoxia-inducible factor prolyl-hydroxylase domain (HIF-PHD) inhibitor that is being investigated for the treatment of anemia secondary to CKD. Methods: A phase2a, multicenter, randomized, double-blind, placebo-controlled, dose-ranging trial (NCT01381094) was undertaken in adults with anemia secondary to CKD stage 3 or 4. Eligible subjects were evenly randomized to 5 groups: $240,370,500$, or $630 \mathrm{mg}$ of once-daily oral vadadustat or placebo for 6 weeks. All subjects received low-dose supplemental oral iron ( $50 \mathrm{mg}$ daily). The primary endpoint was the mean absolute change in hemoglobin $(\mathrm{Hb})$ from baseline to the end of treatment. Secondary endpoints included iron indices, safety, and tolerability. Results: Ninety-three subjects were randomized. Compared with placebo, vadadustat significantly increased $\mathrm{Hb}$ after 6 weeks in a dose-dependent manner (analysis of variance; $p<0.0001$ ). Vadadustat increased the total iron-binding capacity and decreased concentrations of ferritin and hepcidin. The proportion of subjects with at least 1 treatment-emergent adverse event was similar be-
\end{abstract}

\section{KARGER}

E-Mail karger@karger.com www.karger.com/ajn
This article is licensed under the Creative Commons Attribution NonCommercial-NoDerivatives 4.0 International License (CC BYNC-ND) (http://www.karger.com/Services/OpenAccessLicense) Usage and distribution for commercial purposes as well as any distribution of modified material requires written permission. tween vadadustat- and placebo-treated groups. No significant changes in blood pressure, vascular endothelial growth factor, C-reactive protein, or total cholesterol were observed. Limitations of this study included its small sample size and short treatment duration. Conclusions: Vadadustat increased $\mathrm{Hb}$ levels and improved biomarkers of iron mobilization and utilization in patients with anemia secondary to stage 3 or 4 CKD. Global multicenter, randomized phase 3 trials are ongoing in non-dialysis-dependent and dialysis-dependent patients.

(c) 2017 The Author(s)

Published by S. Karger AG, Basel

\section{Introduction}

Patients with chronic kidney disease (CKD) frequently experience anemia, with estimated prevalence rates exceeding $50 \%$ among patients with CKD stage 4 or 5 in the United States. Numerous epidemiological studies [1-3] and post hoc analyses of randomized trials $[4,5]$ support associations between anemia and higher rates of death and cardiovascular disease, as well as poorer quality of life, with significant correlations remaining after adjustment is made for known confounders [6].

Trial registration: ClinicalTrials.gov trial number: NCT01381094.
Emil M. deGoma, MD

Akebia Therapeutics Inc.

245 First Street Suite 1400

Cambridge, MA 02142 (USA)

E-Mail edegoma@akebia.com 
Recombinant human erythropoietin (EPO) and its analogs, collectively termed erythropoiesis-stimulating agents (ESAs), are a mainstay of the management of anemia secondary to CKD $[7,8]$. When administered intravenously or subcutaneously, ESAs stimulate brisk erythropoiesis by achieving supraphysiologic serum EPO levels. While effective in treating anemia, ESAs exhibited cardiovascular-related safety signals after attempts to normalize hemoglobin $(\mathrm{Hb})$ concentrations in a series of large randomized trials [4, 9-11]. Higher ESA doses and the inability to achieve target $\mathrm{Hb}$ levels have been implicated in the adverse outcomes observed with ESA use [5, $12,13]$.

Given the substantial burden of morbidity and mortality facing CKD patients, safe and effective, orally available therapies would be a welcome addition to the therapeutic armamentarium for patients with renal anemia. Stabilization of hypoxia-inducible factor (HIF) via prolyl-hydroxylase domain (PHD) inhibition represents a potentially promising new therapeutic approach to treat anemia secondary to CKD [14-16]. Activation of HIF orchestrates a coordinated response to promote erythropoiesis through the stimulation of endogenous EPO production, increased uptake of iron, and mobilization of iron stores [14].

Vadadustat (AKB-6548) is an oral small molecule HIF-PH inhibitor that is in development for the treatment of anemia in patients with nondialysis-dependent (NDD)-CKD or dialysis-dependent (DD)-CKD. Phase 1 trials have demonstrated dose-proportional pharmacokinetics and dose-dependent increases in serum EPO levels in healthy subjects administered oral vadadustat [17]. In a phase 2a single-ascending-dose study, NDD-CKD subjects treated with vadadustat achieved moderate and physiologic elevations in serum EPO [17]. An open-label, phase $2 \mathrm{a}$ trial showed significant increases in $\mathrm{Hb}$ from baseline after 28 consecutive days of vadadustat administration [18]. In this study, we report the results of a phase 2a trial of vadadustat administered for 6 weeks in patients with anemia secondary to CKD stage 3 or 4 .

\section{Methods}

\section{Trial Design}

This multicenter, 6-week, randomized, double-blind, placebocontrolled, dose-ranging phase 2 a trial evaluated the pharmacodynamic response, safety, and tolerability of once-daily oral dosing of vadadustat in patients with anemia secondary to CKD stage 3 or 4 (ClinicalTrials.gov identifier: NCT01381094). The trial was conducted from June 2011 to February 2012 in compliance with International Conference on Harmonisation of Technical Requirements for Registration of Pharmaceuticals for Human Use-Good Clinical Practice guidelines and the Declaration of Helsinki. The protocol was approved by the Institutional Review Board of each participating institution. All trial patients provided written informed consent.

\section{Trial Population}

Patients were 18-79 years of age with CKD stage 3 or 4 , as defined by the estimated glomerular filtration rate of 30-59 or 15-29 $\mathrm{mL} / \mathrm{min} / 1.73 \mathrm{~m}^{2}$ (not yet on dialysis and not expected to start dialysis within 3 months), respectively, calculated using the Modification of Diet in Renal Disease equation. Eligible patients had not used ESA therapy in the past 11 weeks. All patients had a screening $\mathrm{Hb} \leq 10.5 \mathrm{~g} / \mathrm{dL}$, ferritin $\geq 50 \mathrm{ng} / \mathrm{mL}$, and transferrin saturation (TSAT) $\geq 20 \%$. Patients were excluded if they had anemia attributable to non-CKD causes, including hemolysis, active bleeding or blood loss, or myelodysplastic syndrome, or if they received androgen therapy in the 21 days prior to study dosing or intravenous (IV) iron supplementation exceeding $250 \mathrm{mg}$ in the 21 days prior to the screening visit. Further exclusion criteria included patients who were seropositive for human immunodeficiency virus, hepatitis $\mathrm{B}$ or hepatitis $\mathrm{C}$ virus, or who had cardiovascular disease (defined as one of the following: uncontrolled hypertension; New York Heart Association Class III or IV congestive heart failure; or recent myocardial infarction, acute coronary syndrome, or stroke).

\section{Interventions}

Patients meeting eligibility criteria were randomized to one of 5 groups: vadadustat $240,370,500$, or $630 \mathrm{mg}$ once daily or placebo. Central randomization was performed and stratified by CKD stage and the presence or absence of diabetes mellitus. Patients self-administered the trial medication once daily, regardless of food intake, for 6 weeks ( 42 consecutive days). Trial visits included screening (conducted a maximum of 21 days prior to the baseline visit), baseline (day 1), weeks 1, 2, 4, and 6 (end-of-treatment visit), and a follow-up visit 2 weeks after the last dose of trial medication.

Dosing was discontinued if $\mathrm{Hb}$ levels exceeded $12.5 \mathrm{~g} / \mathrm{dL}$, or if $\mathrm{Hb}$ levels increased by $>2 \mathrm{~g} / \mathrm{dL}$ from the pre-dose average (defined as the average of the 2 values obtained prior to dosing at the screening and baseline visits). A one-time dose reduction was permitted for any patient if $\mathrm{Hb}$ levels increased to $>12.0$ but $\leq 12.5 \mathrm{~g} / \mathrm{dL}$, or if $\mathrm{Hb}$ levels increased by $\geq 1.5$ but $\leq 2.0 \mathrm{~g} / \mathrm{dL}$ from the pre-dose average.

All patients received iron supplementation at a minimum dose of $50 \mathrm{mg}$ of oral elemental iron per day. Patients already receiving iron supplementation (oral or IV) as part of their treatment plan were allowed to continue their regimen with a minimum of the therapeutic equivalent of $50 \mathrm{mg}$ elemental oral iron daily. Any individual IV iron dose could not exceed $250 \mathrm{mg}$.

\section{Trial Endpoints}

The primary endpoint was the mean absolute change between the pre-dose average $\mathrm{Hb}$ and the $\mathrm{Hb}$ measured at the end of the 6 -week treatment period. For patients who underwent dose reduction during the trial, end-of-treatment $\mathrm{Hb}$ was defined as the $\mathrm{Hb}$ value immediately preceding dose reduction. Imputation was not performed for missing values, and missing values were excluded from analysis.

Secondary endpoints included the percentage change in $\mathrm{Hb}$ values from baseline to end of treatment and the absolute and percentage change from baseline in hematocrit, red blood cell (RBC) count,

Vadadustat for Anemia in NDD-CKD 
absolute reticulocyte count, iron indices (iron, total iron binding capacity [TIBC], ferritin, TSAT), and hepcidin. Safety endpoints included the incidence and severity of adverse events (AEs) and serious AEs (SAEs), safety laboratory measures (serum chemistry, hematology, cystatin C, C-reactive protein [CRP], and vascular endothelial growth factor [VEGF]). Serum EPO levels were obtained at baseline and prior to daily study drug dosing at weeks 2 and 6.

Efficacy and safety assessments, including laboratory measures and $\mathrm{AE}$ monitoring, were performed at screening, baseline (day 1), weeks 1, 2, 4, 6 (end of treatment), and during the follow-up visit ( 2 weeks after the last dose of trial medication). All laboratory measures were performed at a central laboratory (except for the urine pregnancy test performed at baseline).

Statistical Analyses

Target enrollment for the trial was 100 patients with CKD stage 3 or 4 .

The intent-to-treat (ITT) population included all randomized patients who received at least one dose of the trial medication. The ITT population was used for analyses of demographics, baseline characteristics, and safety endpoints.

The modified ITT (mITT) population included all randomized patients in the ITT population who had pre-dose efficacy measures ( $\mathrm{Hb}$ and $\mathrm{RBC}$ at both screening and baseline visits) and at least one post-baseline measurement. The mITT population was used for all efficacy endpoint analyses.

The per-protocol (PP) population included all patients in the mITT population who had a trial medication compliance of $\geq 80 \%$ and had no major protocol deviations. The PP population was used for sensitivity analyses.

Comparison of primary efficacy endpoints between treatment groups was performed using a one-way analysis of variance (ANOVA), with change in $\mathrm{Hb}$ level from pre-dose average to end of treatment as the dependent variable and treatment group as the independent variable. Dunnett's test was used to control type 1 experiment-wise error for all treatment comparisons against placebo [19]. With the planned sample size of 90 patients ( 20 enrolled patients per treatment group with $10 \%$ dropout), a Hb difference between any vadadustat treatment group and placebo of $\geq 0.6 \mathrm{~g} / \mathrm{dL}$ could be detected with $85 \%$ power at a significance level of 0.05 , assuming a standard deviation of $0.6 \mathrm{~g} / \mathrm{dL}$ within each group.

Secondary efficacy endpoints were analyzed in both the mITT and $\mathrm{PP}$ populations using a 2 -tailed, paired $t$ test (if the distribution was normal) or a Wilcoxon signed-rank test (if the distribution was not normal) with a significance level of 0.05 .

All AEs were coded using the current version of the Medical Dictionary for Regulatory Activities (version 13.0) and assigned to a system organ class, and the relationship between AEs and trial drug was also recorded. Clinical laboratory assessments (hematology and CRP) and biomarkers (VEGF and cystatin C) were summarized by visit.

\section{Results}

\section{Patient Disposition and Characteristics}

A total of 191 patients were screened and 93 patients randomized into this trial. Of the 93 patients randomized,
91 (98\%) comprised the ITT (safety-evaluable) population (online suppl. Fig. S1; for all online suppl. material, see www.karger.com/doi/10.1159/000464476). Within the ITT population, 89 patients comprised the mITT (efficacy-evaluable) population, and 83 patients comprised the PP (sensitivity) population.

The mean compliance with trial drug, as assessed by returned capsule count, was $>90 \%$ in all treatment groups. No patients discontinued trial treatment due to an excessive $\mathrm{Hb}$ response. Among vadadustat-treated patients, a total of $9(13 \%)$ patients underwent a one-time, protocolspecified dose reduction in study drug due to increased Hb levels ( 2 patients in the $240 \mathrm{mg}$ treatment group, 2 patients in the $500 \mathrm{mg}$ treatment group, and 5 in the $630 \mathrm{mg}$ treatment group). None of these dose reductions were associated with an $\mathrm{AE}$.

Baseline demographics and disease characteristics were generally well balanced among the 5 treatment groups (Table 1). Overall, $75 \%$ of patients had stage $4 \mathrm{CKD}$, the mean (SD) age was 66 (10) years, and there was no difference in gender participation.

\section{Primary Endpoint}

The mean pre-dose average $\mathrm{Hb}$ was $9.77 \mathrm{~g} / \mathrm{dL}$ across all treatment groups, and $\mathrm{Hb}$ levels were similar across the treatment groups (Table 1). Vadadustat significantly increased $\mathrm{Hb}$ in a dose-dependent manner across all doses compared with placebo (Fig. 1a). After 6 weeks of treatment, the $630 \mathrm{mg}$ dose group produced a mean (SD) $\mathrm{Hb}$ increase of 1.39 (0.67) g/dL (Fig. 1a; online suppl. Table S1), with $78 \%$ of patients achieving a $\mathrm{Hb}$ increase $\geq 1 \mathrm{~g} / \mathrm{dL}$ (Fig. 1b). A one-way ANOVA test showed a statistically significant $\mathrm{Hb}$ increase for treatment groups compared with placebo $(p<0.0001)$. Sensitivity analysis showed comparable results in the PP population (online suppl. Table S2).

\section{Secondary and Additional Endpoints}

a. Hematologic parameters over time: treatment with vadadustat 500 and $630 \mathrm{mg}$ achieved statistically significant increases from baseline in mean $\mathrm{Hb}$ levels by week 2 (Fig. 2). All groups treated with vadadustat achieved statistically significant increases from baseline in mean $\mathrm{Hb}$ levels at weeks 4 and 6. Changes in hematocrit and RBC count were consistent with $\mathrm{Hb}$ effects (online suppl. Table S3). Treatment with vadadustat 370,500 , and $630 \mathrm{mg}$ achieved statistically significant increases in absolute reticulocyte count by week 1 (online suppl. Table S3). Peak reticulocytosis occurred at week 2 . At week 6 , there was no significant difference from baseline in absolute reticulocyte count in any treatment group. 


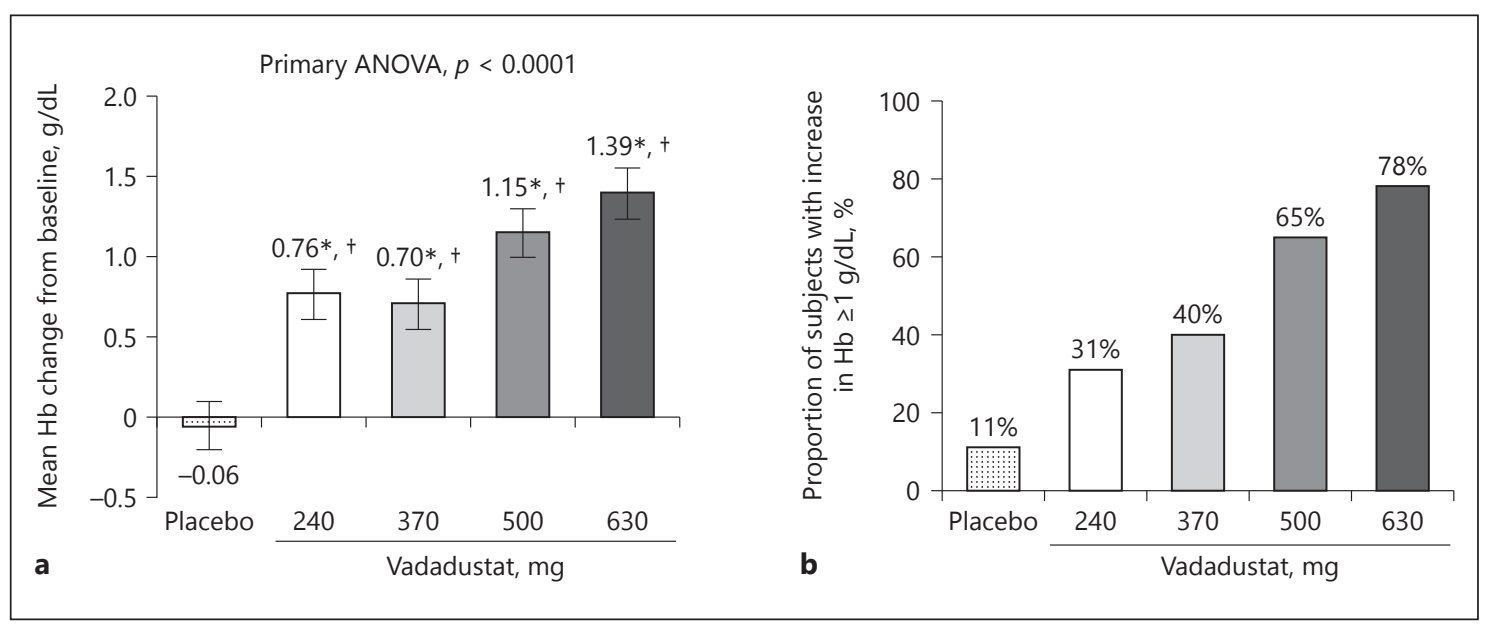

Fig. 1. Effect of trial treatment on hemoglobin $(\mathrm{Hb})$ at week 6 (modified intent to treat population). For patients who underwent dose reduction during the trial, end-of-treatment $\mathrm{Hb}$ was defined as the $\mathrm{Hb}$ immediately preceding dose reduction. Five $(26 \%)$ of the patients in the $630 \mathrm{mg}$ vadadustat treatment group, $2(12 \%)$ of patients in the $500 \mathrm{mg}$ vadadustat treatment group and 2 (11\%) of patients in the $240 \mathrm{mg}$ group underwent protocoldefined dose reductions by week 4 . Imputation was not per- formed for missing values, and missing values were excluded from analysis. a Mean changes in $\mathrm{Hb}( \pm \mathrm{SEM})$ compared to baseline. A one-way analysis of variance test was performed with change from baseline as the dependent variable and treatment group as the independent variable. ${ }^{*} p<0.05$ for comparisons with baseline. ${ }^{\dagger} p<0.05$ for comparisons with placebo. $\mathbf{b}$ Proportion of trial patients who achieved increases in $\mathrm{Hb} \geq 1 \mathrm{~g} / \mathrm{dL}$ at week 6 compared with baseline.

Table 1. Baseline patient characteristics (ITT and mITT populations)

\begin{tabular}{|c|c|c|c|c|c|c|}
\hline \multirow[t]{2}{*}{ Characteristic } & \multicolumn{5}{|c|}{ Vadadustat } & \multirow[t]{2}{*}{ Placebo } \\
\hline & $240 \mathrm{mg}$ & $370 \mathrm{mg}$ & $500 \mathrm{mg}$ & $630 \mathrm{mg}$ & Total & \\
\hline Safety population (ITT), $n$ & 18 & 18 & 17 & 19 & 72 & 19 \\
\hline Age, years & $64.2(12.2)$ & $68.9(7.8)$ & $64.7(9.5)$ & $64.9(8.8)$ & $65.7(9.7)$ & $64.9(10.0)$ \\
\hline Women & $9(50)$ & $7(39)$ & $13(77)$ & $7(37)$ & $36(50)$ & $12(63)$ \\
\hline \multicolumn{7}{|l|}{ Race } \\
\hline Black/African American & $6(33)$ & $4(22)$ & $4(24)$ & $7(37)$ & $21(29)$ & $3(16)$ \\
\hline White/Caucasian & $10(56)$ & $13(72)$ & $13(77)$ & $9(47)$ & $45(63)$ & $14(74)$ \\
\hline Other & $2(11)$ & $1(6)$ & $0(0)$ & $3(16)$ & $6(8)$ & $2(11)$ \\
\hline Weight, kg & $84.5(18.4)$ & $84.4(17.1)$ & $83.9(18.3)$ & $86.2(23.2)$ & $84.8(19.1)$ & $81.9(20.9)$ \\
\hline BMI, $\mathrm{kg} / \mathrm{m}^{2}$ & $30.6(4.2)$ & $29.8(5.6)$ & $32.1(6.8)$ & $29.1(6.5)$ & $30.3(5.8)$ & $29.8(6.6)$ \\
\hline $\mathrm{eGFR}, \mathrm{mL} / \mathrm{min} / 1.73 \mathrm{~m}^{2}$ & $22.3(12.1)$ & $25.0(11.2)$ & $25.3(9.2)$ & $24.9(12.3)$ & $24.4(11.1)$ & $25.2(11.1)$ \\
\hline \multicolumn{7}{|l|}{ CKD stage } \\
\hline 3 & $5(28)$ & $4(22)$ & $4(24)$ & $5(26)$ & $18(25)$ & $5(26)$ \\
\hline 4 & $13(72)$ & $14(78)$ & $13(77)$ & $14(74)$ & $54(75)$ & $14(74)$ \\
\hline Diabetes mellitus & $14(78)$ & $12(67)$ & $12(71)$ & $13(68)$ & $51(71)$ & $13(68)$ \\
\hline History of ESA use & $6(33)$ & $6(33)$ & $4(24)$ & $2(11)$ & $18(25)$ & $7(37)$ \\
\hline Efficacy population (mITT), $n$ & 18 & 16 & 17 & 19 & 70 & 19 \\
\hline $\mathrm{Hb}, \mathrm{g} / \mathrm{dL}$ & $9.47(0.80)$ & $9.96(0.68)$ & $9.96(0.64)$ & $9.70(0.81)$ & $9.76(0.75)$ & $9.86(0.69)$ \\
\hline TIBC, $\mu \mathrm{g} / \mathrm{dL}$ & $219.4(39.4)$ & $249.9(55.4)$ & $243.6(45.4)$ & $231.8(36.2)$ & $236.0(45.2)$ & $243.1(40.0)$ \\
\hline Ferritin, ng/mL & $349.5(226.3)$ & $257.8(213.2)$ & $322.8(312.2)$ & $240.2(142.1)$ & $291.4(228.7)$ & $200.8(116.0)$ \\
\hline Hepcidin, ng/mL & $326.3(222.0)$ & $241.0(158.9)$ & $251.1(112.4)$ & $282.8(239.3)$ & $276.8(191.3)$ & $258.3(144.1)$ \\
\hline
\end{tabular}

ITT, intent-to-treat; mITT, modified ITT; Hb, hemoglobin; BMI, body mass index; CKD, chronic kidney disease; eGFR, estimated glomerular filtration rate; ESA, erythropoiesis-stimulating agent; TIBC, total iron binding capacity. Values are $n$ (\%) or mean (SD), as applicable. 


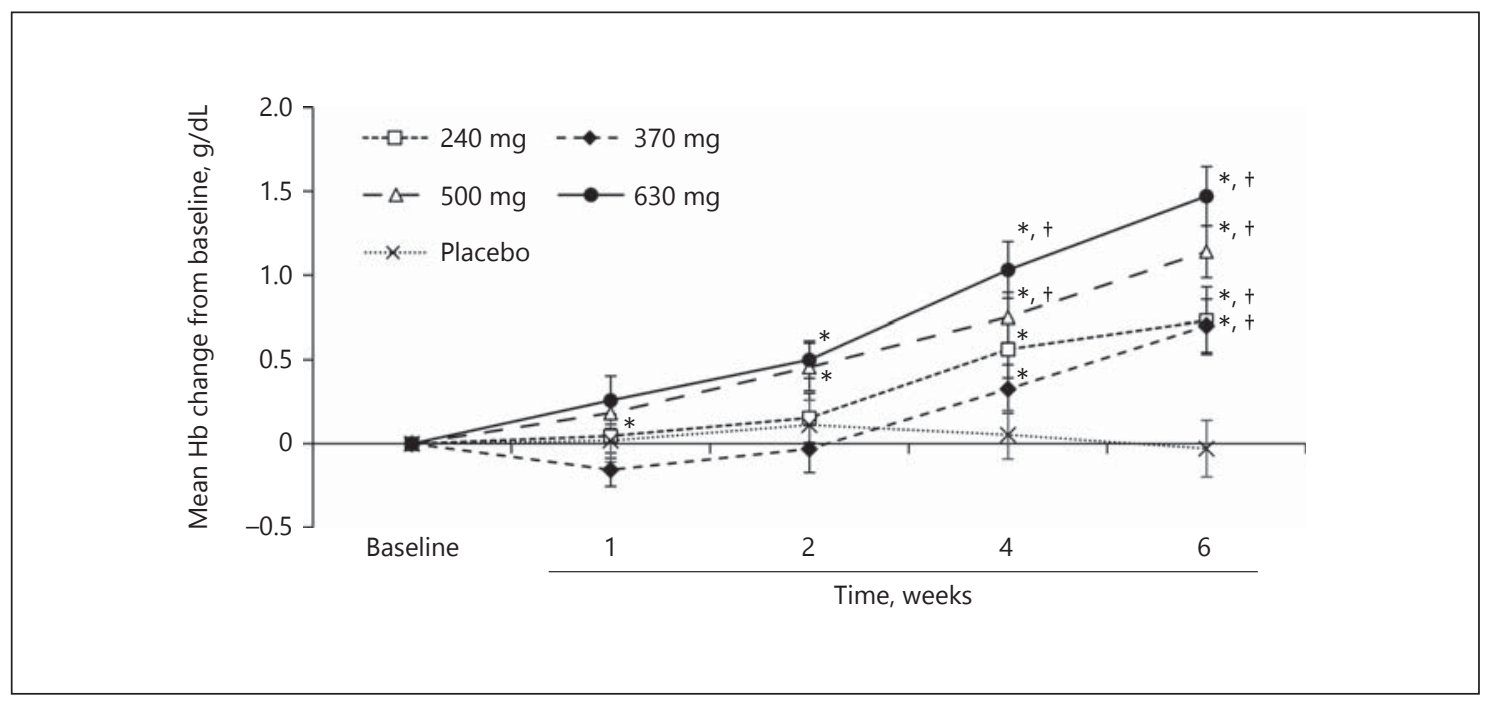

Fig. 2. Observed mean hemoglobin $(\mathrm{Hb})$ concentration over the trial period during administration of vadadustat or placebo (modified intent to treat population). Data are expressed as the mean \pm SEM Hb value at each time point. ${ }^{*} p<0.05$ for comparisons with baseline. ${ }^{\dagger} p<0.05$ for comparisons with placebo.

b. Measures of iron utilization: significant decreases in ferritin levels $(p<0.05)$ and increases in TIBC $(p<0.05)$ were observed for all vadadustat treatment groups at weeks 2, 4, and 6 (Fig. 3a, b). Treatment with vadadustat significantly decreased hepcidin by $90-139 \mathrm{ng} / \mathrm{mL}(34-$ $39 \%$ ) from baseline to week 6 at doses of 370,500 , and 630 $\mathrm{mg}(p<0.05$; Fig. 3c).

\section{Safety}

The proportion of patients with one or more AEs was similar between treatment groups (overall, $47.2 \%$ in the vadadustat groups and $57.9 \%$ in the placebo group; online suppl. Table S4), and was distributed evenly across the dosing groups, with no apparent dose-related effect. Of the 102 AEs reported, 97 (95.1\%) were mild or moderate and 5 (4.9\%) were assessed as severe but unrelated to treatment. The most commonly reported AEs consisted of gastrointestinal disorders, which occurred with a similar frequency between trial arms (12 [16.7\%] for the vadadustat treatment groups versus 3 [15.8\%] for the placebo group respectively). Among vadadustat treatment groups, there was no evidence of a relationship between the dose and the rate of AEs.

Ten vadadustat-treated patients (13.9\%) reported 15 AEs that were assessed as treatment-related; these were mild $(n=13)$ or moderate $(n=2)$ in severity and exhibited no dose dependency (online suppl. Table S4). The most commonly reported AEs that were considered treatment-related were gastrointestinal disorders $(n=7)$; all other AEs did not cluster by organ system.
Seven SAEs were reported in 7 (9.7\%) vadadustattreated patients and $1(5.3 \%)$ in placebo-treated patients. No individual SAE was experienced by more than 1 patient and no SAEs were considered related to trial medication. One patient in the $370 \mathrm{mg}$ vadadustat treatment group died from sustained ventricular tachycardia and cardiac arrest during hospitalization for severe azotemia due to CKD progression. This SAE was considered to be unrelated to trial medication.

Four patients $(5.6 \%)$ in the vadadustat treatment groups were withdrawn from the trial due to AEs. Besides the previously discussed case of severe azotemia, the other withdrawals included 1 patient for severe coronary artery disease with non-ST elevated myocardial infarction (unrelated to trial medication), 1 patient for nausea (mild, probably related to trial medication), and 1 patient for palpitations (mild, probably related to trial medication) and worsening of hypertension (possibly related to trial medication).

There were no trends in vital signs, electrocardiographic parameters, or safety laboratory values, and the number of patients with recorded abnormal values were similar in each treatment group. At week 6, no significant changes from baseline were observed in systolic or diastolic blood pressure, CRP, VEGF, cystatin $\mathrm{C}$, or total cholesterol in any of the treatment groups. Pre-dose serum EPO levels obtained prior to vadadusat dosing at weeks 2 and 6 were similar to baseline values. 
Fig. 3. Observed mean ferritin concentration and total iron binding capacity (TIBC) over the trial period during administration of vadadustat or placebo (modified intent to treat population). Data are expressed as the mean \pm SEM at each time point. a Mean change in ferritin concentration $( \pm$ SEM $)$ compared to baseline. b Mean change in TIBC compared to baseline. c Mean change in hepcidin concentration compared to baseline. ${ }^{*} p<0.05$ for comparisons with baseline. ${ }^{\dagger} p<0.05$ for comparisons with placebo.

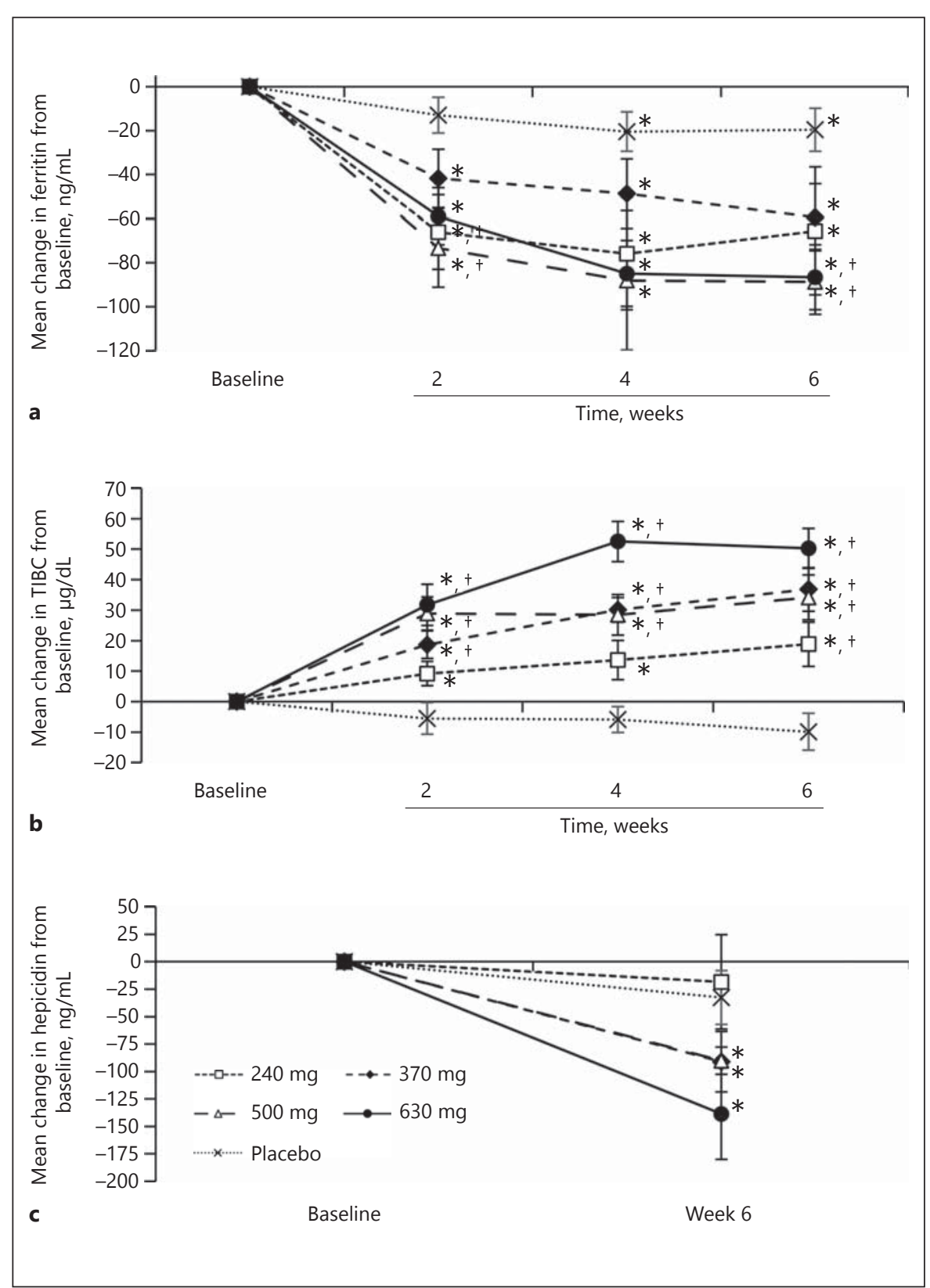

\section{Discussion}

This phase 2a trial demonstrated the ability of vadadustat - an orally administered HIF-PHD inhibitor currently in development for the treatment of anemia - to increase $\mathrm{Hb}$ levels in a dose-dependent manner over 6 weeks in patients with anemia secondary to CKD stage 3 or 4 , with an overall incidence of AEs comparable to placebo.

HIF stabilization via PHD inhibition promotes erythropoiesis by stimulating endogenous EPO secretion and improving iron availability [20]. Prior studies of vadadustat in healthy volunteers and CKD patients demonstrated modest increases in endogenous EPO levels - which were similar to those observed following exposure to moderate altitude [21] - as well as preserved physiologic diurnal variation of serum EPO levels $[17,18]$. In this study, the evaluation of serum EPO levels was restricted to pre-dose testing to confirm whether levels return to baseline within $24 \mathrm{~h}$ of vadadustat administration, consistent with the normal physiologic state. Our results in- 
dicate that pre-dose serum EPO levels were not increased compared with baseline prior to vadadustat dosing at weeks 2 and 6 . These data are consistent with trials completed in healthy subjects and NDD-CKD patients in which serum EPO levels following vadadustat administration increased and returned to baseline within $24 \mathrm{~h}$, avoiding supraphysiologic EPO elevations and maintaining the normal EPO diurnal variation [17]. In the current trial, vadadustat decreased hepcidin and ferritin and increased TIBC, consistent with improved iron mobilization and utilization. The reticulocyte profile mimicked the predicted response to altitude-associated hypoxia [22]. Similar results were observed in a 20 -week, phase $2 \mathrm{~b}$ trial of vadadustat administered to patients with anemia secondary to NDD-CKD [23].

In this 6-week study, no safety signals were noted in vadadustat-treated patients. There were no changes in clinic systolic or diastolic blood pressures following 6 weeks of vadadustat treatment. Although several studies in both animal models and humans have demonstrated a positive correlation between HIF stabilization and levels of VEGF, markers of inflammation [24], or cholesterol [25-27], raising the concern for potential tumor progression or increased atherothrombotic risk, there were no changes in VEGF, CRP, or total cholesterol levels noted in the current trial. These data suggest that vadadustat elicits a targeted erythropoietic effect, a hypothesis to be tested in larger, longer clinical trials and non-clinical, mechanistic studies. Since the completion of this trial, two additional phase 2 trials, one in patients with NDD-CKD (NCT01906489) [23] and one in patients with DD-CKD (NCT02260193) [28, 29], have evaluated the effect of vadadustat when administered for up to 20 weeks. Four global, randomized phase 3 trials are ongoing to compare the efficacy and safety of anemia treatment with vadadustat versus that with darbepoetin alfa in patients with anemia secondary to DD-CKD (INNO ${ }_{2}$ VATE: NCT02865850, NCT02892149) and NDD-CKD(PRO 2 TECT:NCT02680574,NCT02648347).

Limitations of this phase $2 \mathrm{a}$ trial include its modest sample size and short duration of treatment. The trial was not designed to assess titration or maintenance of target $\mathrm{Hb}$, nor was the trial powered for safety. In addition, inclusion criteria for $\mathrm{Hb}$ and ferritin were established prior to the 2011 FDA modification of ESA product labeling [30]. The revised labels recommend a $\mathrm{Hb}$ threshold for ESA initiation below $10 \mathrm{~g} / \mathrm{dL}$ and ferritin of at least $100 \mathrm{ng} / \mathrm{mL}$ prior to ESA initiation.

Preclinical and clinical studies conducted to date with vadadustat have identified several potential favorable at- tributes for this HIF-PHI. Flexibility of dosing is supported by results in which vadadustat administered once daily or three times weekly maintained stable $\mathrm{Hb}$ levels in DD-CKD patients following conversion from epoetin alfa [31]. Key pharmacokinetic parameters (half-life, maximum concentration, area under the curve) of vadadustat have been consistent across healthy volunteers (Caucasian or Japanese), NDD-CKD, and DD-CKD populations, indicating no clinically meaningful effect of CKD severity or ethnicity on the pharmacokinetic profile $[23,32,33]$. The hemodialysis procedure has no clinically meaningful effect on serum levels of vadadustat indicating that vadadustat can be dosed without regard to the patient's dialysis schedule [34]. In vitro studies of vadadustat have revealed no effect on cytochrome P450 (CYP) 3A4 activity (data not shown), and a clinical drug-drug interaction study showed no effect of vadadustat on the pharmacokinetics of celecoxib, a model substrate for CYP 2C9 [35]. As a result, there is a low likelihood of drug-drug interactions with commonly prescribed drugs in this patient population (e.g., atorvastatin, losartan). Finally, as described above, the administration of vadadustat in clinical trials did not alter serum levels of VEGF, cholesterol, or triglycerides [23, 31].

In summary, in this 6-week randomized, placebo-controlled, phase 2a trial, vadadustat increased $\mathrm{Hb}$ levels and was well tolerated in an initial cohort of patients with anemia secondary to CKD stage 3 or 4 .

\section{Acknowledgments}

The authors thank K. Jones (formerly of Akebia), K. Manzur (medical writer at Lenimen Consulting, Inc.) and Lucid Partners Ltd. for editorial assistance with this manuscript, J. Baker (formerly of Pharm-Olam International, Ltd.) for statistical analysis help, R. Shalwitz and C. Hartman and C. Flinn (formerly of Akebia) for trial design, execution, and monitoring support and assistance with data analysis and interpretation of trial results, and $\mathrm{R}$. Farzaneh-Far (formerly of Akebia) for assistance with data analysis and interpretation of trial results. The authors also thank all patients and their families, as well as investigators, research nurses, trial coordinators, and other site staff who participated in this trial.

\section{Contributions}

All authors, except those of the Sponsor, contributed patients to the trial. All authors had full access to the trial data and the analyses. Each author contributed important intellectual content during manuscript drafting or revision and all authors reviewed the manuscript and signed off for its accuracy.
386

Am J Nephrol 2017;45:380-388 DOI: $10.1159 / 000464476$
Martin/Smith/Maroni/Zuraw/deGoma 
EMD assumes responsibility for this trial: that it has been reported honestly, accurately, and transparently; that no important aspects of the trial have been omitted; and that any discrepancies from the trial as planned (and, if relevant, registered) have been explained.

\section{Support}

Akebia Therapeutics, Inc., sponsored and funded this trial.

\section{Financial Disclosures}

E.M.D., Q.C.Z., and B.J.M. are employees of Akebia Therapeutics, Inc. and hold stock and/or stock options in Akebia Therapeutics, Inc. E.R.M. and M.T.S. were trial investigators and were paid for their services by Akebia Therapeutics, Inc.; however, they were not compensated as authors of the manuscript and they declare having no other competing financial or other interests relevant to this work.

\section{References}

1 Jones M, Ibels L, Schenkel B, Zagari M: Impact of epoetin alfa on clinical end points in patients with chronic renal failure: a meta-analysis. Kidney Int 2004;65:757767.

2 Locatelli F, Pisoni RL, Combe C, Bommer J, Andreucci VE, Piera L, Greenwood R, Feldman HI, Port FK, Held PJ: Anaemia in haemodialysis patients of five European countries: association with morbidity and mortality in the dialysis outcomes and practice patterns study (DOPPS). Nephrol Dial Transplant 2004;19:121-132.

3 Regidor DL, Kopple JD, Kovesdy CP, Kilpatrick RD, McAllister CJ, Aronovitz J, Greenland S, Kalantar-Zadeh K: Associations between changes in hemoglobin and administered erythropoiesis-stimulating agent and survival in hemodialysis patients. J Am Soc Nephrol 2006;17:11811191.

4 Besarab A, Bolton WK, Browne JK, Egrie JC, Nissenson AR, Okamoto DM, Schwab SJ, Goodkin DA: The effects of normal as compared with low hematocrit values in patients with cardiac disease who are receiving hemodialysis and epoetin. N Engl J Med 1998;339: 584-590.

5 Szczech LA, Barnhart HX, Inrig JK, Reddan DN, Sapp S, Califf RM, Patel UD, Singh AK: Secondary analysis of the CHOIR trial epoetin-alpha dose and achieved hemoglobin outcomes. Kidney Int 2008;74:791798.

6 Servilla KS, Singh AK, Hunt WC, Harford AM, Miskulin D, Meyer KB, Bedrick EJ, Rohrscheib MR, Tzamaloukas AH, Johnson HK, Zager PG: Anemia management and association of race with mortality and hospitalization in a large not-for-profit dialysis organization. Am J Kidney Dis 2009;54:498510.

7 Locatelli F, Barany P, Covic A, De Francisco A, Del Vecchio L, Goldsmith D, Horl W, London G, Vanholder R, Van Biesen W; ERA-EDTA ERBP Advisory Board: Kidney Disease: Improving Global Outcomes guidelines on anaemia management in chronic kidney disease: a European renal best practice position statement. Nephrol Dial Transplant 2013;28:1346-1359.
8 Kidney Disease: Improving Global Outcomes (KDIGO) Anemia Work Group: KDIGO clinical practice guideline for anemia in chronic kidney disease. Kidney Int Suppl 2012;2:279-335.

9 Singh AK, Szczech L, Tang KL, Barnhart H, Sapp S, Wolfson M, Reddan D; CHOIR Investigators: Correction of anemia with epoetin alfa in chronic kidney disease. $\mathrm{N}$ Engl J Med 2006;355:2085-2098.

10 Drueke TB, Locatelli F, Clyne N, Eckardt KU, Macdougall IC, Tsakiris D, Burger HU, Scherhag A; CREATE Investigators: Normalization of hemoglobin level in patients with chronic kidney disease and anemia. N Engl J Med 2006;355:20712084.

11 Pfeffer MA, Burdmann EA, Chen CY, Cooper ME, de Zeeuw D, Eckardt KU, Feyzi JM, Ivanovich $\mathrm{P}$, Kewalramani R, Levey AS, Lewis EF, McGill JB, McMurray JJ, Parfrey P, Parving HH, Remuzzi G, Singh AK, Solomon SD, Toto R; TREAT Investigators: A trial of darbepoetin alfa in type 2 diabetes and chronic kidney disease. N Engl J Med 2009;361:20192032.

12 McCullough PA, Barnhart HX, Inrig JK, Reddan D, Sapp S, Patel UD, Singh AK, Szczech LA, Califf RM: Cardiovascular toxicity of epoetin-alfa in patients with chronic kidney disease. Am J Nephrol 2013;37:549558.

13 Fishbane S, Besarab A: Mechanism of increased mortality risk with erythropoietin treatment to higher hemoglobin targets. Clin J Am Soc Nephrol 2007;2:1274-1282.

14 Lenihan CR, Winkelmayer WC: The dawning of a new day in CKD anemia care? J Am Soc Nephrol 2016;27:968-970.

15 Semenza GL: Regulation of oxygen homeostasis by hypoxia-inducible factor 1 . Physiology (Bethesda) 2009;24:97-106.

16 Safran M, Kaelin WG Jr: HIF hydroxylation and the mammalian oxygen-sensing pathway. J Clin Invest 2003;111:779-783.

17 Buch A, Maroni BJ, Hartman CS: Dose exposure relationship of vadadustat is independent of the level of renal function. J Am Soc Nephrol 2015;26:\#SA-PO537.

18 Hartman C, Smíth MT, Flinn C, Shalwitz I, Peters KG, Haase VH: AKB-6548, a new hy- poxia-inducible factor prolyl hydroxylase inhibitor increases hemoglobin while decreasing ferritin in a 28 -day, phase 2 a dose escalation study in stage 3 and 4 chronic kidney disease patients with anemia. J Am Soc Nephrol 2011;22:435A.

19 Dunnett CW: A multiple comparison procedure for comparing several treatments with a control. J Am Stat Assoc 1955;50:10961121.

20 Maxwell PH, Eckardt KU: HIF prolyl hydroxylase inhibitors for the treatment of renal anaemia and beyond. Nat Rev Nephrol 2016; $12: 157-168$

21 Windsor JS, Rodway GW: Heights and haematology: the story of haemoglobin at altitude. Postgrad Med J 2007;83:148-151.

22 Klausen T, Poulsen TD, Fogh-Andersen N, Richalet JP, Nielsen OJ, Olsen NV: Diurnal variations of serum erythropoietin at sea level and altitude. Eur J Appl Physiol Occup Physiol 1996;72:297-302.

23 Pergola PE, Spinowitz BS, Hartman CS, Maroni BJ, Haase VH: Vadadustat, a novel oral HIF stabilizer, provides effective anemia treatment in nondialysis-dependent chronic kidney disease. Kidney Int 2016;90:11151122.

24 Gao L, Chen Q, Zhou X, Fan L: The role of hypoxia-inducible factor 1 in atherosclerosis. J Clin Pathol 2012;65:872-876.

$25 \mathrm{Li}$, Thorne LN, Punjabi NM, Sun CK, Schwartz AR, Smith PL, Marino RL, Rodriguez A, Hubbard WC, O'Donnell CP, Polotsky VY: Intermittent hypoxia induces hyperlipidemia in lean mice. Circ Res 2005;97: 698-706.

26 Ramakrishnan SK, Taylor M, Qu A, Ahn $\mathrm{SH}$, Suresh MV, Raghavendran K, Gonzalez FJ, Shah YM: Loss of von HippelLindau protein (VHL) increases systemic cholesterol levels through targeting hypoxia-inducible factor $2 \alpha$ and regulation of bile acid homeostasis. Mol Cell Biol 2014;34: 1208-1220.

27 Jun J, Reinke C, Bedja D, Berkowitz D, Bevans-Fonti S, Li J, Barouch LA, Gabrielson K, Polotsky VY: Effect of intermittent hypoxia on atherosclerosis in apolipoprotein E-deficient mice. Atherosclerosis 2010;209:381386

Vadadustat for Anemia in NDD-CKD 
28 Haase VH, Chertow G, Block G, Pergola P, Zuraw Q, Khawaja Z, Sharma A, Maroni BJ, McCullough PA: Vadadustat Maintains Hemoglobin (Hb) Levels in Dialysis-Dependent Chronic Kidney Disease (DD-CKD) Patients Independent of Systemic Inflammation or Prior Dose of Erythropoiesis-Stimulating Agent (ESA): Kidney Week. Chicago, American Society of Nephrology, 2016, TH-PO960. http:// www.akebia.com/wp-content/themes/ akebia/img/media-kit/abstracts-posterspresentations/20161117\%20Ph2\%200011\%20 ASN\%202016_FINAL_v4_09Nov16.pdf.

29 Hartman CS, Farmer TM, Annis K, Kazazi F, Pollack P, Shalwitz R: Phase 2 Study of AKB6548, A Novel Hypoxia-Inducible Factor Prolyl-Hydroxylase Inhibitor (HIF-PHI) in Patients with End Stage Renal Disease (ESRD) Undergoing Hemodialysis (HD). Philadelphia, Kidney Week, 2014, INFO25. http:// akebia.com/wp-content/themes/akebia/img/ media-kit/abstracts-posters-presentations/ 20141106_Akebia_ASN_Informational_ Poster-FINAL.pdf.

30 Center for Drug Evaluation Research: FDA Drug Safety Communication: Modified Dos- ing Recommendations to Improve the Safe Use of Erythropoiesis-Stimulating Agents (ESAs) in Chronic Kidney Disease. Silver Spring, US Food and Drug Administration, 2011.

31 Haase VH, Hartman CS, Maroni B, Farzaneh-far R, McCullough PA: Vadadustat, A Novel, Oral Treatment for Anemia of CKD, Maintains Stable Hemoglobin Levels in Dialysis Patients Converting from Erythropoiesis-Stimulating Agents: National Kidney Foundation Spring Clinical Meeting. Boston, The National Kidney Foundation, 2016, abstr 171. http://akebia.com/wp-content/themes/ akebia/img/media-kit/abstracts-posterspresentations/Akebia_NKF 2016 Poster_ FINAL.pdf.

32 Buch A, Maroni B, Hartman C: Dose exposure relationship of vadadustat is independent of the level of renal function. J Am Soc Nephrol 2015;26:747A.

33 Chandorkar GA, Jhee S, Han D, Schmelzer K, Sherman M, Manzur K, Sharma A, Zuraw Q: Pharmacokinetics, Pharmacodynamics, and Safety of Single and Multiple Oral Doses of Vadadustat in Healthy Japanese and Cau- casian Subjects: American Society of $\mathrm{Ne}$ phrology's Kidney Week. Chicago, American Society of Nephrology, 2016, SA-PO506. 34 Buch A, Farmer TM, Hartman C, Alcorn H, Shalwitz R: Hemodialysis has minimal impact on the pharmacokinetics of AKB-6548, a once-daily oral inhibitor of hypoxia-inducible factor prolyl-hydroxylases (HIF-PHs) for the treatment of anemia related to chronic kidney disease (CKD). In: ASN Kidney Week. Philadelphia, PA; 2014, FR-PO952. http://akebia.com/wp-content/themes/ akebia/img/media-kit/abstracts-posterspresentations/20141106_Akebia_ASN_PK_ Poster-FINAL.pdf. (accessed December 12, 2016).

35 Chandorkar GA, Farzaneh-Far R, Buch A, Maroni B: A Drug-Drug Interaction Study to Evaluate the Effect of Vadadustat on the Pharmacokinetics of Celecoxib - a CYP2C9 Substrate - in Healthy Volunteers: ERA-EDTA Congress. Vienna, ERA-EDTA, 2016, SP322. http:// akebia.com/wp-content/themes/akebia/img/ media-kit/abstracts-posters-presentations/ 20160523\%20ERA-EDTA\%20Poster_FINAL. pdf. 